Research Article

\title{
On the Circumference of 3-Connected Cubic Triangle-Free Plane Graphs
}

\author{
Adthasit Sinna $(\mathbb{D}$, Witthawas Phanthawimol $\mathbb{D}$, and Sirirat Singhun $(\mathbb{D}$ \\ Department of Mathematics, Faculty of Science, Ramkhamhaeng University, Bangkok 10240, Thailand \\ Correspondence should be addressed to Adthasit Sinna; adthasit@rumail.ru.ac.th
}

Received 2 June 2021; Revised 27 July 2021; Accepted 2 August 2021; Published 14 August 2021

Academic Editor: M. M. Bhatti

Copyright ( 2021 Adthasit Sinna et al. This is an open access article distributed under the Creative Commons Attribution License, which permits unrestricted use, distribution, and reproduction in any medium, provided the original work is properly cited.

The circumference of a graph $G$ is the length of a longest cycle in $G$, denoted by $\operatorname{cir}(G)$. For any even number $n$, let $c(n)=\min$ $\{\operatorname{cir}(G) \mid G$ is a 3-connected cubic triangle-free plane graph with $n$ vertices\}. In this paper, we show that an upper bound of $c(n)$ is $n+1-3\lfloor n / 136\rfloor$ for $n \geq 136$.

\section{Introduction}

Graph theory has applications in various research areas. In chemistry and biology, it gives an illustration of molecular structures in $[1,2]$, and it identifies interactions between humans and nature in [3]. Graph theory can be combined together with other branches of mathematics such as algebraic graph theory, matroid theory, graph labeling, and topological graph theory. Recently, it is combined with fixed point theory in $[4,5]$.

We consider only simple undirected graph. Let $G=$ ( $V(G), E(G)$ ) be a graph, where $V(G)$ is the vertex set of $G$ and $E(G)$ is the edge set of $G$. The degree of a vertex $v$ is the number of edges incident to $v$. A graph is cubic if all vertices have degree exactly three. A plane graph is a graph embedded in a plane without edge crossing. An $x y$-path of a graph $G$ is a sequence of distinct vertices $x=v_{0}, v_{1}, v_{2}, \ldots$ $v_{n}=y$ of $G$ such that $v_{i} v_{i+1} \in E(G)$, for all $i \in\{0,1,2, \ldots$, $n-1\}$. Similar to $x y$-path, a cycle of a graph $G$ is defined as a sequence of distinct vertices $u_{1}, u_{2}, \ldots, u_{m}, u_{1}$ of $G$ such that $u_{m} u_{1}, u_{j} u_{j+1} \in E(G)$, for all $j \in\{1,2, \ldots, m-1\}$. The triangle is a cycle of length 3 , and a graph is triangle-free if it contains no triangle. A graph $G$ is connected if there is a $u v$-path for all distinct $u, v \in V(G)$. A graph $G$ is $k$-connected if $|V(G)| \geq k$, and for any $X \subseteq V(G)$ of size $k-1$, a graph $G-X$ is connected. A $k$-leg fragment $H$ is a connected plane graph such that there are exactly $k$ vertices of $H$ with degree two, and the remaining vertices have degree three. A Hamilton cycle of a graph $G$ is a cycle containing all vertices of $G$.

Finding Hamilton cycles in graphs is a famous topic in graph theory. In 1880, Tait [6] conjectured that a 3-connected cubic plane graph has a Hamilton cycle. Tutte [7] constructed the Tutte fragment, a triangle-free 3-leg fragment with 15 vertices, and showed the following statement.

Lemma 1 (see [7]). Let $T$ be the Tutte fragment in Figure 1. Then,

(i) There is an $x y$-path (respectively, an $x z$-path) containing all vertices of $H$, and

(ii) For each yz-path $P,|V(P)| \leq 14$, i.e., $P$ misses at least one vertex from $T$.

Using three Tutte fragments and one vertex, the Tutte graph is the first counterexample of Tait's conjecture with 46 vertices. Later, Holton and McKay [8] showed that a smallest counterexample of this conjecture is the Barnette-Horák-Lederburg graph, a 38-vertex triangle-free graph containing two Tutte fragments. Moreover, Grinberg graph [9] and Faulkner-Younger graph [10] are other counterexamples of this conjecture without any Tutte fragment.

For 4-connected graphs embedded in surfaces, Tutte [11] proved that every 4-connected plane graph has a Hamilton 


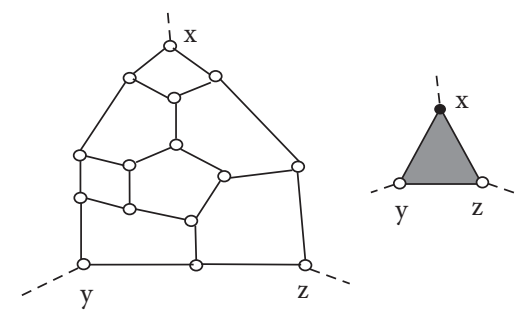

Figure 1: The Tutte fragment.

cycle. Extending Tutte's result, Thomas and Yu [12] proved that every 4-connected projective plane graph has a Hamilton cycle. In the case of toroidal graph, Grünbaum [13] and Nash-Williams [14] independently conjectured that every 4connected toroidal graph has a Hamilton cycle. For the closest result of this conjecture, Thomas and $\mathrm{Yu}$ [15] proved that every 5-connected toroidal graph has a Hamilton cycle. A closed knight's tour on the chessboard is an example of Hamilton cycle problem. Some results of a closed knight's tour are shown in $[16,17]$.

Define the circumference of a graph $G$ as the length of a longest cycle in a graph $G$, denoted by $\operatorname{cir}(G)$. Since Tait's conjecture is false, the generalized question is what is the minimum circumference of any 3-connected cubic (triangle-free) plane graph with $n$ vertices? Then, let $b(n)=\min \{\operatorname{cir}(G) \mid G$ is a 3-connected cubic plane graph with $n$ vertices $\}$ and $c(n)=\min \{\operatorname{cir}(G) \mid G$ is a 3 -connected cubic triangle-free plane graph with $n$ vertices\}.

Note that $b(n) \leq c(n)$, and $n$ must be even because the sum of degrees of all vertices is even. For the exact value of $c(n)$, Holton and McKay [8] showed that $b(n)=c(n)=n$ if $n \leq 36$, and $b(38)=c(38)=37$. Note that $c(n)$ is still unknown for $n \geq 40$.

For a lower bound of $b(n)$, Jackson [18] showed that an $n$-vertex 3-connected cubic graph has circumference $\Omega\left(n^{0.694}\right)$ for sufficiently large $n$. Bilinski et al. [19] improved this to $\Omega\left(n^{0.753}\right)$, and then, Liu et al. [20] improved to $\Omega\left(n^{0.8}\right)$. This implies $b(n) \geq \Omega\left(n^{0.8}\right)$ for sufficiently large $n$.

For an upper bound of $b(n)$ for all even numbers $n \leq 38$. Let $\left\{G_{i}\right\}$ be the sequence of graphs such that $G_{1}$ is the the Barnette-Horák-Lederburg graph, and $G_{i+1}$ is obtained from $G_{i}$ by replacing all vertices of $G_{i}$ by triangle. Then, $G_{i}$ is a $38\left(3^{i-1}\right)$-vertex graph with circumference $37\left(3^{i-1}\right)$. Note that a longest cycle of $G_{i}$ misses $3^{i-1}$ vertices. Let $H$ be a graph obtained by $G_{i}$ by replacing $k$ vertices of $G_{i}$ by triangle, where $k<\left|V\left(G_{i}\right)\right|$. Then, $\operatorname{cir}(H) \leq|V(H)|-3^{i-1}$. This gives $b(n) \leq n-3^{i-1}$ if $38\left(3^{i-1}\right) \leq n \leq 38\left(3^{i}\right)-2$.

For a triangle-free graph, Lu [21] constructed a $\left[(4 k+7)\left(4 k^{\prime}+2\right)\right]$-vertex graph, where $k \geq 2$ and $k^{\prime} \geq 1$, with circumference $(4 k+7)\left(4 k^{\prime}+2\right)-\min$ $\left\{4 k+8,4 k^{\prime}-2\right\}$. If $k^{\prime}=k+3$, then this graph implies $c(n) \leq n-\sqrt{n+49 / 4}+5 / 2$ for infinitely many integers $n$. Next, Lo and Schmidt [22] constructed a $122 m$-vertex graph with circumference at most $120 \mathrm{~m}$ for some positive integer $m$. This implies $c(n) \leq 60 n / 61$ for some $n$ which are divisible by 122 .
In this paper, we show that an upper bound of $c(136)$ is 133 in Theorem 1, and the graph $G(k)$, a $136 k$-vertex graph, has the circumference $133 k+1$ in Lemma 4 . In Theorem 2, we improve an upper bound of $c(n)$ to $n+1-3\lfloor n / 136\rfloor$, for all $n \geq 138$.

\section{Main Results}

First, we construct the 4-leg fragment $H$ with 136 vertices as in Figure 2(a). Note that $H$ is triangle-free and contains nine Tutte fragments. Next, for any positive integer $i$, we let $H_{i}$ be a copy of $H$ such that $a_{i}, b_{i}, c_{i}$, and $d_{i}$ correspond to $a, b, c$, and $d$ in $H$, respectively. Finally, we construct a graph $G(k)$ from the union of graphs $H_{1}, H_{2}, \ldots, H_{k}$, by adding edges $b_{k} a_{1}, d_{k} c_{1}, b_{i} a_{i+1}$, and $d_{i} c_{i+1}$, for all $i \in\{1,2, \ldots, k-1\}$, as in Figure 3. Note that $G(k)$ is a 3-connected cubic triangle-free plane graph with $136 k$ vertices.

By the construction of $G(k)$, a longest cycle $C$ of $G(k)$, where $k \geq 2$, must pass through some fragments $H_{i}$. Since $H_{i}$ is a 4-leg fragment, $C \cap H_{i}$ are either an $x y$-path, where $x$, $y \in\left\{a_{i}, b_{i}, c_{i}, d_{i}\right\}$, or a union of two disjoint paths where all end vertices of these paths are in $\left\{a_{i}, b_{i}, c_{i}, d_{i}\right\}$. In order to find the circumference of $G(k)$, it is necessary to show the maximum size of each path of $H$ in Lemma 2 and of each union of two disjoint paths of $H$ in Lemma 3.

Lemma 2. Let $H$ be the 4-leg fragment in Figure 2(a). Then,

(i) Each ab-path in $H$ contains at most 130 vertices

(ii) Each ac-path (respectively, bd-path) in H contains at most 132 vertices

(iii) Each ad-path (respectively, bc-path) in H contains at most 133 vertices

(iv) Each cd-path in $H$ contains at most 119 vertices

Proof. Let $M$ be the graph obtained from $H$ by contracting all Tutte fragments into vertices $A_{1}, A_{2}, A_{3}, B_{1}, B_{2}, B_{3}, C_{1}, C_{2}$, and $C_{3}$ as in Figure 2(b). Note that $|V(M)|=10$ and, the vertices $a$, $b, c$, and $d$ of $H$ belong to the fragments represented by $A_{3}, B_{2}$, $C_{2}$, and $C_{3}$, respectively. Let $P$ be a longest $x y$-path, where $x, y \in\{a, b, c, d\}$, and let $Q$ be a path of $M$ corresponding to $P$. So, $Q$ is the $u v$-path, where $u, v \in\left\{A_{3}, B_{2}, C_{2}, C_{3}\right\}$. Note that if $Q$ passes through all vertices in $V(M)-\{w\}$, then $|V(H)-V(P)| \leq 10$, i.e., $P$ may miss the vertex $w$ and one vertex from each Tutte fragments. Otherwise, $\mid V(H)-$ $V(P) \mid \geq 15$. Then, $|V(Q)-\{w\}|$ should be the maximum in $M$. Thus, we consider four cases as follows.

Case 1: $P$ is an $a b$-path. Then, $Q$ is an $A_{3} B_{2}$-path. If $w \in Q$, then either $C_{2} \in Q$ or $C_{3} \in Q$. Suppose $w \in Q$. Since there is only one $A_{3} B_{2}$-path missing only vertex $w$, the sequence of vertices of $Q$ is $A_{3}, A_{1}, A_{2}, C_{2}, C_{1}, C_{3}$, $B_{3}, B_{1}$, and $B_{2}$. This implies $P$ passes through each fragment in this order. By Lemma 1, $P$ misses vertex $w$ and other five vertices from the fragments $A_{1}, B_{1}, C_{1}$, $C_{2}$, and $C_{3}$. Then, $|V(P)|=130$. 


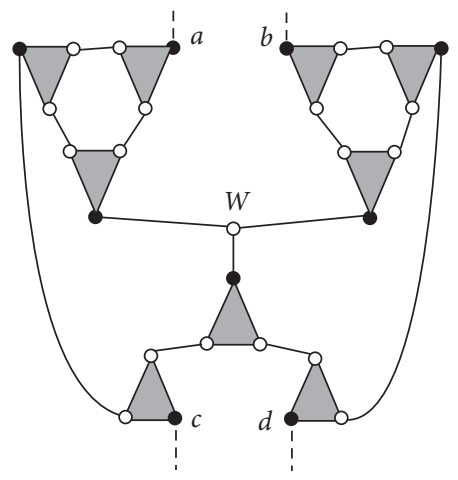

(a)

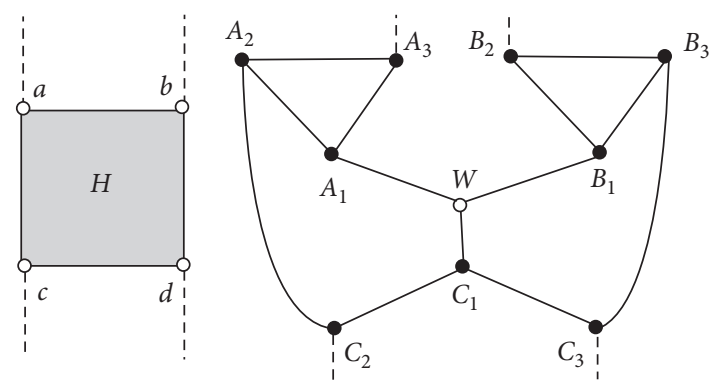

(b)

Figure 2: (a) The 4-leg fragment $H$. (b) The graph $M$ obtained from $H$ by contracting all Tutte fragments into vertices.

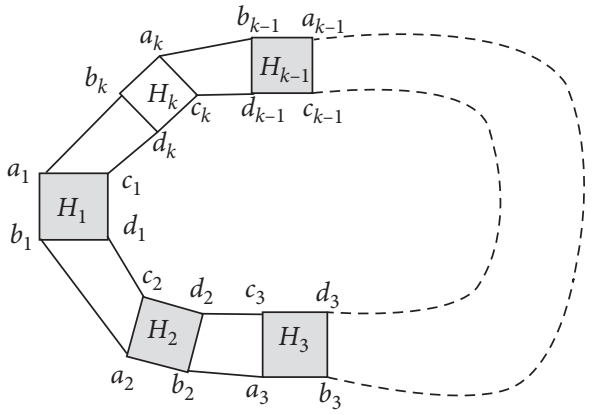

FIgURE 3: The graph $G(k)$.

Case 2: $P$ is an ac-path, or a $b d$-path. By the symmetry of $H$, we consider only ac-path. Since there is only one $A_{3} C_{2}$-path containing all vertices of $M$, the sequence of vertices of $Q$ is $A_{3}, A_{2}, A_{1}, w, B_{1}, B_{2}, B_{3}, C_{3}, C_{1}$, and $C_{2}$. By Lemma 1, $P$ misses four vertices from the fragments $A_{2}, B_{2}, C_{1}$, and $C_{3}$. Then, $|V(P)|=132$.

Case 3: $P$ is an $a d$-path, or a $b c$-path. By the symmetry of $H$, we consider only ad-path. Since there is only one $A_{3} C_{3}$-path containing all vertices of $M$, the sequence of vertices of $Q$ is $A_{3}, A_{1}, A_{2}, C_{2}, C_{1}, w, B_{1}, B_{2}, B_{3}$, and $C_{3}$. By Lemma 1, $P$ misses three vertices from the fragments $A_{1}, B_{2}$, and $C_{2}$. Then, $|V(P)|=133$.

Case 4: $P$ is a $c d$-path. Then, $Q$ is a $C_{2} C_{3}$-path. If $C_{1} \in Q$, then either $\left\{A_{1}, A_{2}, A_{3}\right\} \subseteq Q$ or $\left\{B_{1}, B_{2}\right.$, $\left.B_{3}\right\} \subseteq Q$. Suppose $C_{1} \in Q$. Since there is only one $C_{2} C_{3}$-path missing only vertex $C_{1}$, the sequence of vertices of $Q$ is $C_{2}, A_{2}, A_{3}, A_{1}, w, B_{1}, B_{2}, B_{3}$, and $C_{3}$. Note that $Q$ misses the vertex $C_{1}$ so $P$ misses 15 vertices from fragment $C_{1}$. By Lemma $1, P$ misses other two vertices from the fragments $A_{3}$ and $B_{2}$. Then, $|V(P)|=119$.

For a union of two disjoint paths, we define a ( $w x$, $y z$ )-dual path as the disjoint union of a $w x$-path and a $y z$-path. Note that the intersection of an $a d$-path and a $b c$-path in $H$ is not empty. By the symmetry of $H$, there are exactly two cases of $(w x, y z)$-dual path, where $\{w, x, y, z\}=$ $\{a, b, c, d\}$ in $H$ as follows.
Lemma 3. Let $H$ be the 4-leg fragment in Figure 2(a). Then,

(i) Each $(a b, c d)$-dual path in $H$ contains at most 133 vertices

(ii) Each $(a c, b d)$-dual path in $H$ contains at most 134 vertices

Proof. Let $M$ be the graph defined from $H$ in Figure 2(b). Let $D$ be the largest $(w x, y z)$-dual path in $H$ such that $\{w, x, y, z\}=\{a, b, c, d\}$. Let $D_{M}$ be the dual path corresponding to $D$. Without loss of generality, we consider two cases as follows:

Case 1: $D$ is an $(a b, c d)$-dual path. Then, $D_{M}$ is an $\left(A_{3} B_{2}, C_{2} C_{3}\right)$-dual path. Since there is exactly one dual path containing all vertices of $M$, the sequences of these two paths of $D_{M}$ are $A_{3}, A_{2}, A_{1}, w, B_{1}, B_{3}, B_{2}$, and $C_{2}$, $C_{1}, C_{3}$, respectively. By Lemma $1, D$ misses three vertices from the fragments $A_{2}, B_{3}$, and $C_{1}$. Then, $|V(D)|=133$.

Case 2: $D$ is an $(a c, b d)$-dual path. Then, $D_{M}$ is an $\left(A_{3} C_{2}, B_{2} C_{3}\right)$-dual path. Without the loss of generality, there is only one dual path containing all vertices of $M$. Then, the sequences of these two paths of $D_{M}$ are $A_{3}$, $A_{2}, A_{1}, w, C_{1}, C_{2}$, and $B_{2}, B_{1}, B_{3}, C_{3}$, respectively. By Lemma $1, D$ misses two vertices from the fragments $A_{2}$ and $B_{1}$. Then, $|V(D)|=134$.

Since we obtain Lemma 2 and Lemma 3, we have $c(136) \leq 133$ by showing the following theorem.

Theorem 1. There is a 136-vertex 3-connected cubic trianglefree plane graph with circumference 133.

Proof. Let $K$ be a graph obtained from the fragment $H$ by adding edges $a c$ and $b d$ (see Figure $4(a)$ ). Note that $K$ is a 3connected cubic triangle-free plane graph with 136 vertices. Let $M^{\prime}$ be the graph obtained from $K$ by contracting all Tutte fragments into vertices $A_{1}, A_{2}, A_{3}, B_{1}, B_{2}, B_{3}, C_{1}, C_{2}$, and $C_{3}$ as in Figure 4(b).

Let $C$ be a longest cycle of $K$ satisfying the condition in each following case. 


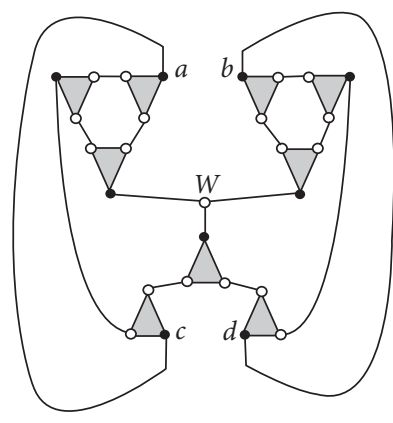

(a)

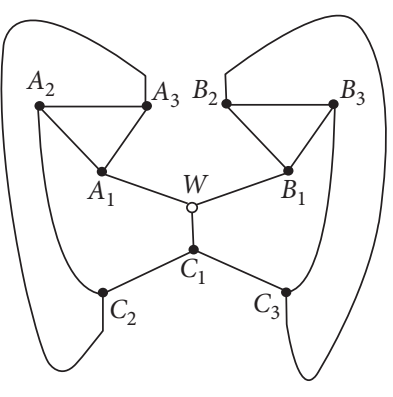

(b)

Figure 4: (a) The graph $K$. (b) The graph $M^{\prime}$ obtained from $K$ by contracting all Tutte fragments into vertices.

Case 1. Neither $a c$ nor $b d$ is in $C$. Let $D$ be the cycle of $M^{\prime}$ corresponding to $C$. Then, neither $A_{3} C_{2}$ nor $B_{2} C_{3}$ is in $D$. If $w \in V(D)$, then either $V(D)=\left\{A_{1}, A_{2}, A_{3}\right\}$ or $V(D)=$ $\left\{B_{1}, B_{2}, B_{3}\right\}$. Suppose $w \in V(D)$. Since there is only one cycle containing all vertices of $M^{\prime}$, the sequence of vertices of $D$ is $w, A_{1}, A_{3}, A_{2}, C_{2}, C_{1}, C_{3}, B_{3}, B_{2}, B_{1}$, and $w$. By Lemma 1 , $C$ misses five vertices from the fragments $A_{3}, B_{2}, C_{1}, C_{2}$, and $C_{3}$. Then, $|V(C)|=131$.

Case 2. Only one edge from $\{a c, b d\}$ is in $C$. Assume without loss of the generality that $a c \in E(C)$, but $b d \in E(C)$. Then, $C-\{a c\}$ is an $a c$-path in $H$. By Lemma 2 (ii), $|V(C)|=\mid V$ $(C-\{a c\}) \mid=132$.

Case 3. Both $a c$ and $b d$ are in $C$. Then, $C-\{a c, b d\}$ is an $(a b, c d)$-dual path in $H$. By Lemma $3(\mathrm{i}),|V(C)|=\mid V$ $(C-\{a c, b d\}) \mid=133$.

Comparing the length of $C$ in each case, we conclude that $\operatorname{cir}(K)=133$.

Recall that a graph $G(k)$ has $136 k$ vertices. Then, we give the circumference of $G(k)$ as follows.

Lemma 4. The circumference of $G(k)$ is $133 k+1$ for all positive integers $k$.

Proof. For $k=1$, we use the similar proof to Theorem 1 to show that $\operatorname{cir}(G(1))=134$. Suppose $k \geq 2$. Let $C$ be a longest cycle of $G(k)$ passing through all fragments $H_{1}, H_{2}, H_{3}, \ldots$, $H_{k}$. Without loss of generality, we generate each case by the possible cases of $\mathrm{C} \cap \mathrm{H}_{1}$ as follows.

Case 1: $C \cap H_{1}$ is an $x y$-path, where $x \in\left\{a_{1}, c_{1}\right\}$ and $y \in\left\{b_{1}, d_{1}\right\}$.

Then, $C \cap H_{i}$ is an $x y$-path, where $x \in\left\{a_{i}, c_{i}\right\}$ and $y \in\left\{b_{i}, d_{i}\right\}$, for any $i \in\{2,3, \ldots, k\}$ (see Figure 5(a)). By Lemma $2, C \cap H_{i} \leq 133$. This implies $|V(C)| \leq 133 k$.

Case 2: $C \cap H_{1}$ is either a $b_{1} d_{1}$-path or an $a_{1} c_{1}$-path. Assume without loss of generality that $C \cap H_{1}$ is a $b_{1} d_{1}$-path. Then, $C \cap H_{k}$ is an $a_{k} c_{k}$-path, and $C \cap H_{i}$ is an $\left(a_{i} b_{i}, c_{i} d_{i}\right)$-dual path, for any $i \in\{2,3, \ldots, k-1\}$ (see Figure 5(b)). By Lemma 2(ii) and Lemma 3(i), $|V(C)|=2(132)+133(k-2)=133 k-2$.
Case 3: $C \cap H_{1}$ is an $\left(a_{1} c_{1}, b_{1} d_{1}\right)$-dual path. Then, $C \cap H_{i}$ is an $\left(a_{i} b_{i}, c_{i} d_{i}\right)$-dual path, for any $i \in\{2,3, \ldots$, $k\}$. Note that $b_{k} a_{1}, d_{k} c_{1}, b_{i} a_{i+1}, d_{i} c_{i+1} \in E(C)$, for all $i=1,2, \ldots, k-1$ (see Figure 5(c)). By Lemma 3, $|V(C)|=134+133(k-1)=133 k+1$.

For the case that a longest cycle $D$ does not intersect some fragments $H_{i}$, we can assume, without loss of generality, that $D \cap H_{i} \neq \varnothing$ if $1 \leq i \leq m$, otherwise $D \cap H_{i}=\varnothing$ (see Figure 5(d)). This case is similar to Case 2, so we can show that $V(D)=2(132)+133(m-2)=133 m-2 \leq$ $133 k-2$.

Then, we conclude that a longest cycle in $G(k)$ is a cycle defined in Case 3 which implies that $\operatorname{cir}(G(k))=133 k$ +1 .

The ladder, denoted by $L_{m}$, is a graph with $V\left(L_{m}\right)=$ $\left\{u_{i}, v_{i} \mid i=1,2, \ldots, m\right\}$ and $E\left(L_{m}\right)=\left\{u_{i} \quad v_{i} \mid i=1,2, \ldots\right.$, $m\} \cup\left\{u_{i} u_{i+1}, v_{i} v_{i+1} \mid i=1,2, \ldots, m-1\right\}$. Then, $L_{m}$ is a triangle-free 4-leg fragment with $2 m$ vertices. It is easy to show the following statements:

(i) There is a $u_{1} v_{1}$-path (respectively, $u_{m} v_{m}$-path) containing all vertices of $L_{m}$

(ii) If $m$ is odd, then there are a longest $u_{1} v_{m}$-path (respectively, $v_{1} u_{m}$-path) containing all vertices of $L_{m}$, and a longest $u_{1} u_{m}$-path (respectively, $v_{1} v_{m}$-path) missing exactly one vertex from $L_{m}$

(iii) If $m$ is even, then there are a longest $u_{1} v_{1}$-path (respectively, $u_{m} v_{m}$-path) containing all vertices of $L_{m}$, and a longest $u_{1} v_{m}$-path (respectively, $v_{1} u_{m}$-path) missing exactly one vertex from $L_{m}$

(iv) For any $x, y, z$ such that $\{x, y, z\}=\left\{v_{1}, u_{m}, v_{m}\right\}$ except for the case $(x, y, z)=\left(v_{m}, v_{1}, u_{m}\right)$, there is a $\left(u_{1} x, y z\right)$-dual path containing all vertices of $L_{m}$

Combining a graph $G(k)$ and a ladder $L_{m}$, we have the main theorem as follows.

Theorem 2. For any even number $n \geq 136$, there is an n-vertex 3-connected cubic triangle-free plane graph with circumference $n+1-3\lfloor n / 136\rfloor$.

Proof. For the case that $n$ is the multiple of 136, it is shown in Lemma 4. Assume that $n$ is not the multiple of 


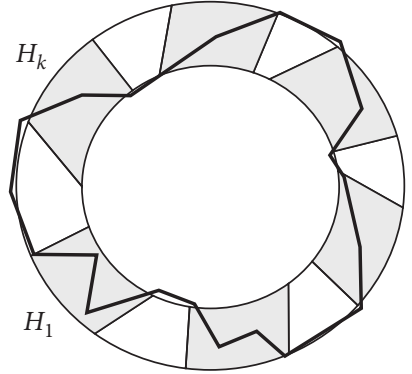

(a)

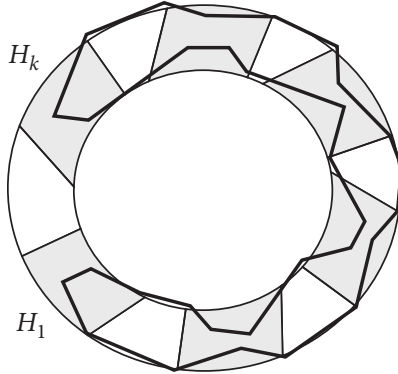

(b)

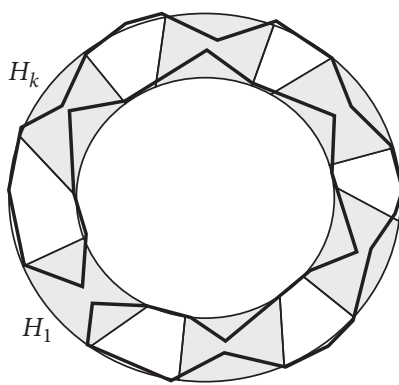

(c)

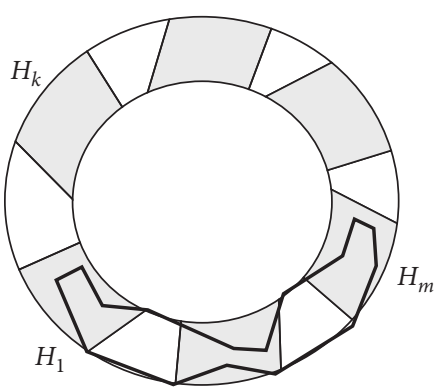

(d)

Figure 5: Cycles in Lemma 4. (a) A cycle $C$ in Case 1. (b) A cycle $C$ in Case 2. (c) A cycle $C$ in Case 3. (d) A cycle $D$ where it does not intersect some fragments $H_{i}$ of $G(k)$.

136. Let $k=\lfloor n / 136\rfloor$ and $m=(n-136 k) / 2$. Let $G$ be an $n$-vertex graph with $V(G)=V\left(G_{k}\right) \cup V\left(L_{m}\right)$ and $E(G)=$ $\left[E\left(G(k)-\left\{b_{1} a_{2}, d_{1} c_{2}\right\}\right] \cup E\left(L_{m}\right) \cup\left\{b_{1} u_{1}, d_{1} v_{1}, u_{m} a_{2}, v_{m} c_{2}\right\}\right.$ (see Figure 6). By this construction, it is easy to show that $G$ is a 3 -connected cubic triangle-free plane graph. Let $C^{*}$ be a longest cycle of $G(k)$. Then, $C^{*}$ is a cycle defined in Case 3 of Lemma 4 which misses $3 k-1$ vertices from $G(k)$. Since $G(k)-\left\{b_{1}\right.$ $\left.a_{2}, d_{1} c_{2}\right\}$ is a subgraph of $G$, a longest cycle of $G$ should miss at least $3 k-1$ vertices, i.e., $\operatorname{cir}(G) \leq n-(3 k-1)$.

Define paths $P: b_{1}, u_{1}, u_{2}, \ldots, u_{m}, a_{2}$, and $Q: c_{1}$, $v_{1}, v_{2}, \ldots, v_{m}, d_{2}$. Let $C^{\prime}=\left[C^{*}-\left\{b_{1} a_{2}, d_{1} c_{2}\right\}\right] \cup P \cup Q$. Then, $C^{\prime}$ is a cycle in $G$ containing $n+1-3 k$ vertices. Hence, $\operatorname{cir}(G)=n+1-3 k$.

\section{Discussion and Open Problems}

It can be implied from Theorem 1 and Theorem 2 that $c(136) \leq 133$, and $c(n) \leq n+1-3\lfloor n / 136\rfloor$ if $n \geq 138$. This improves most of upper bound of $c(n)$ from $[21,22]$ except for some cases in [21]. Then, we will compare ours to that result as follows.

Theorem 3 (see [21]). For any integer $k \geq 2$ and $k^{\prime} \geq 1$, there is a 3-connected cubic triangle-free plane graph with $[(4 k+$ $\left.7)\left(4 k^{\prime}+2\right)\right]$ vertices and circumference $(4 k+7)\left(4 k^{\prime}+2\right)-$ $\min \left\{4 k+8,4 k^{\prime}-2\right\}$.

Putting appropriate $k$ and $k^{\prime}$, this theorem gives a better upper bound of $c(n)$ than ours in the following cases: $c(n) \leq n-2$ if $n \in\{90,114\}, c(n) \leq n-6$ if $n \in\{150,190$, $230,270,310,350,390\}, c(n) \leq n-10$ if $n \in\{210,266,322$, $378,434,490\}, c(n) \leq n-14$ if $n \in\{270,342,414,486,558$, $630\}, \quad c(n) \leq n-16$ if $n \in\{330,390,450,510,570,630$, $690,750,810\}, c(n) \leq n-18$ if $n \in\{418,506,594,682,770$, $858,946\}, \quad c(n) \leq n-20$ if $n \in\{494,570,646,722,798$, $874,950\}, c(n) \leq n-22$ if $n \in\{598,702,806,910,1014\}$, $c(n) \leq n-24$ if $n \in\{690,782,874,966,1058,1150\}, c(n) \leq$ $n-26$ if $n \in\{930,1050,1170\}, c(n) \leq n-28$ if $n \in\{918$, $1026,1134,1242,1350\}, \quad c(n) \leq n-30$ if $n \in\{1054,1190$, $1326,1462\}, \quad c(n) \leq n-32 \quad$ if $n \in\{1178,1320,1462\}$, $c(n) \leq n-34 \quad$ if $\quad n \in\{1330,1482\}, \quad c(n) \leq n-36 \quad$ if $n \in\{1470,1638\}, c(n) \leq n-38$ if $n=1638, c(n) \leq n-40$ if $n=1794$, and $c(n) \leq n-42$ if $n=1978$.

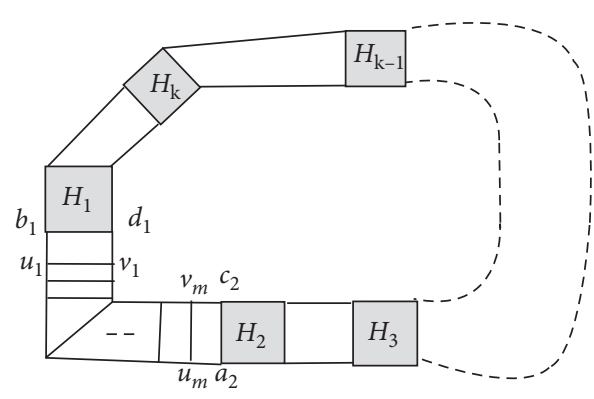

Figure 6: The graph $G$ defined in Theorem 2.

For an open problem, we define $c_{P}(n)$ (respectively, $\left.c_{T}(n)\right)$ as the minimum of $\operatorname{cir}(G)$, where $G$ is a 3-connected cubic triangle-free projective plane graph (respectively, toroidal graph) with $n$ vertices. The Petersen graph, a 10-vertex graph, is a smallest 3-connected cubic triangle-free nonhamiltonian graph which can be embedded in both projective plane and torus. This implies that $c_{P}(n)=c_{T}(n)=n$ if $n \leq 8$, and $c_{P}(10)=c_{T}(10)=9$. Since a plane graph is also a projective plane graph and a toroidal graph, we have the following inequality: $c_{P}(n) \leq c(n)$ and $c_{T}(n) \leq c(n)$. Then, we give the following questions.

Question 1. What is an upper bound of $c_{P}(n)$ ?

Question 2. What is an upper bound of $c_{T}(n)$ ?

\section{Data Availability}

No data were used to support this study.

\section{Conflicts of Interest}

The authors declare that they have no conflicts of interest.

\section{Acknowledgments}

This work was supported by Ramkhamhaeng University. 


\section{References}

[1] R. García-Domenech, J. Gálvez, J. V. de Julián-Ortiz, and L. Pogliani, "Some new trends in chemical graph theory," Chemical Reviews, vol. 108, no. 3, pp. 1127-1169, 2008.

[2] J. Blazewicz, A. Hertz, D. Kobler, and D. de Werra, "On some properties of DNA graphs," Discrete Applied Mathematics, vol. 98, no. 1-2, pp. 1-19, 1999.

[3] S. de Juan, A. Ospina-Álvarez, S. Villasante, and A. Ruiz-Frau, "A Graph Theory approach to assess nature's contribution to people at a global scale," Scientific Reports, vol. 11, no. 1, p. 9118, 2021.

[4] E. Ameer, H. Aydi, M. Arshad, and M. De la Sen, "Hybrid cirić type graphic $(\Upsilon, \wedge)$-contraction mappings with applications to electric circuit and fractional differential equations," Symmetry, vol. 12, no. 3, p. 467, 2020.

[5] N. Mlaiki, N. Souayah, T. Abdeljawad, and H. Aydi, "A new extension to the controlled metric type spaces endowed with a graph," Advances in Difference Equations, vol. 94, 2021.

[6] P. G. Tait, "Listingś topologie," The London, Edinburgh, and Dublin Philosophical Magazine and Journal of Science, vol. 17, no. 103 , pp. $30-46,1884$.

[7] W. T. Tutte, "On Hamiltonian circuits," Journal of the London Mathematical Society, vol. 21, no. 2, pp. 98-101, 1946.

[8] D. A. Holton and B. D. McKay, "The smallest non-Hamiltonian 3-connected cubic planar graphs have 38 vertices," Journal of Combinatorial Theory-Series B, vol. 45, no. 3, pp. 305-319, 1988.

[9] E. J. Grinberg, "Plane homogeneous graphs of degree three without Hamiltonian circuits," Latvian mathematical yearbook, vol. 4, pp. 51-58, 1968.

[10] G. B. Faulkner and D. H. Younger, "Non-Hamiltonian cubic planar maps," Discrete Mathematics, vol. 7, no. 1-2, pp. 67-74, 1974.

[11] W. T. Tutte, "A theorem on planar graphs," Transactions of the American Mathematical Society, vol. 82, no. 1, p. 99, 1956.

[12] R. Thomas and X. X. Yu, "4-Connected projective-planar graphs are Hamiltonian," Journal of Combinatorial TheorySeries B, vol. 62, no. 1, pp. 114-132, 1994.

[13] B. Grünbaum, "Polytopes, graphs, and complexes," Bulletin of the American Mathematical Society, vol. 76, no. 6, pp. 11311202, 1970.

[14] C. St and J. A. Nash-Williams, "Unexplored and semi-explored territories in graph theory," New Directions in Graph Theory, pp. 149-186, Academic Press, New York, NYUSA, 1973.

[15] R. Thomas and X. Yu, "Five-connected toroidal graphs are Hamiltonian," Journal of Combinatorial Theory-Series B, vol. 69, no. 1, pp. 79-96, 1997.

[16] S. Singhun, P. Ruengmanee, and A. Sinna, "A closed-knight's tour on some cylinder chessboards," AKCE International Journal of Graphs and Combinatorics, vol. 17, no. 1, pp. 296-302, 2020.

[17] S. Singhun and N. Loykaew, "Closed knight's tour problem on some $(m, n, k, 1)$-rectangular tubes," Asian-European Journal of Mathematics, vol. 14, no. 6, p. 215094, 2021.

[18] B. Jackson, "Longest cycles in 3-connected cubic graphs," Journal of Combinatorial Theory - Series B, vol. 41, no. 1, pp. 17-26, 1986.

[19] M. Bilinski, B. Jackson, J. Ma, and X. Yu, "Circumference of 3connected claw-free graphs and large Eulerian subgraphs of 3edge-connected graphs," Journal of Combinatorial TheorySeries B, vol. 101, no. 4, pp. 214-236, 2011.
[20] Q. Liu, X. Yu, and Z. Zhang, "Circumference of 3-connected cubic graphs," Journal of Combinatorial Theory-Series B, vol. 128, pp. 134-159, 2018.

[21] X. Lu, "A note on 3-connected cubic planar graphs," Discrete Mathematics, vol. 310, no. 13, pp. 2054-2058, 2010.

[22] O.-H. S. Lo and J. M. Schmidt, "Longest cycles in cyclically 4edge-connected cubic planar graphs," Australasian Journal of Combinatorics, vol. 72, no. 1, pp. 155-162, 2018. 